\title{
A COMPARATIVE ANALYSIS OF ACUTE TOXICITIES IN HYPOFRACTIONATED RADIOTHERAPY VERSUS CONVENTIONAL RADIOTHERAPY IN EARLY-STAGE BREAST CANCER AFTER BREAST CONSERVATION SURGERY
}

\author{
Sukrutha Boggula1', Ashwini Gopal2, Suneetha Mulinti³, Alluri Krishnam Raju ${ }^{4}$
}

${ }^{1}$ Registrar, Department of Radiation Oncology, Basavatarakam Indo-American Cancer Hospital and Research Institute, Hyderabad, Telangana, India.

${ }^{2}$ Registrar, Department of Radiation Oncology, Basavatarakam Indo-American Cancer Hospital and Research Institute, Hyderabad,

Telangana, India.

${ }^{3}$ Consultant Radiation Oncologist, Department of Radiation Oncology, Continental Hospital, Hyderabad, Telangana, India.

${ }^{4}$ Consultant Radiation Oncologist, Department of Radiation Oncology, Basavatarakam Indo-American Cancer Hospital and Research Institute, Hyderabad, Telangana, India.

\section{ABSTRACT}

\section{BACKGROUND}

Adjuvant radiation to early breast cancer who underwent breast conservation can be delivered in two different fractionation schedules.

Aim- Analysis of acute toxicities in hypofractionated radiotherapy versus conventional radiotherapy in early-stage breast cancer.

\section{MATERIALS AND METHODS}

This is a single institutional prospective non-randomised controlled trial done on patients with early breast cancer who underwent breast conservation surgery and required adjuvant radiation therapy in our hospital which is a tertiary care cancer hospital in South India. 40 patients with 20 in each arm, who were treated from June 2014 - January 2016 are included in the study. The reactions were graded according to the RTOG acute toxicity grading criteria. Pearson's Chi-square test was used and a p-value of 0.05 was used for comparison of acute toxicity. Relation of breast volume to the grade of acute toxicity was analysed using ANOVA test.

\section{RESULTS}

This study with patient and tumour characteristics of both treatment groups were similar in age, tumour histology, tumour staging, nodal staging and hormone receptor status. In the conventional group, 15 patients (75\%) had grade-2 reaction, 3 patients (15\%) had grade- 3 reactions, while in HF group most patients had grade- 1 skin reactions (60\%), 8 patients (40\%) had grade- 2 reactions and none of them showed grade- 3 reactions. Analysis of acute toxicity showed statistical significance between both groups ( $p=0.002)$. In the CF group, 3 patients who had grade-3 skin reaction had a mean breast volume of $1484.67 \mathrm{cc}( \pm 167 \mathrm{cc}), 15$ patients who had grade-2 skin reaction had a mean breast volume of $956 \mathrm{cc}( \pm 219)$ and 2 patients who had grade- 1 skin reaction had a mean breast volume of $622 \mathrm{cc}( \pm 125 \mathrm{cc})$. In the HF group, the mean breast volume for 12 patients who had grade-1 skin toxicity is $763.83 \mathrm{cc}( \pm 223)$ and for 8 patients who had grade-2 skin toxicity is $1197.50 \mathrm{cc}( \pm 242.9)$. Higher the breast volume, higher is the grade of acute skin reaction in both the groups.

\section{CONCLUSION}

Hypofractionated radiotherapy can be recommended as a standard treatment after BCS with short duration of treatment and comparable skin toxicities with that of conventional radiotherapy.

\section{KEY WORDS}

Hypofractionation, Early Breast Cancer, Acute Toxicity, Skin Reactions.

HOW TO CITE THIS ARTICLE: Boggula S, Gopal A, Mulinti S, et al. A comparative analysis of acute toxicities in hypofractionated radiotherapy versus conventional radiotherapy in early-stage breast cancer after breast conservation surgery. J. Evolution Med. Dent. Sci. 2018;7(30):3348-3351, DOI: 10.14260/jemds/2018/755

\section{BACKGROUND}

Adjuvant radiation to breast was traditionally delivered over 5 weeks to a dose of 50 Gy in 25 fractions. The newer hypofractionated radiation is delivered as an adjuvant local treatment to early breast cancer patients who have undergone breast conservation surgery.

'Financial or Other Competing Interest': None.

Submission 13-06-2018, Peer Review 08-07-2018,

Acceptance 13-07-2018, Published 21-07-2018.

Corresponding Author:

Sukrutha Boggula,

\#3-17/102, Quli Quttub Shah Colony,

Puppalaguda, Manikonda,

Hyderabad-500089, Telangana, India.

E-mail: sukrutha86b@gmail.com

DOI: $10.14260 /$ jemds $/ 2018 / 755$

\section{(c) $(\mathbf{P})$}

This involves fewer treatments, delivers a higher dose per treatment and shorter overall treatment time. In the present study we compared the acute toxicities between the hypofractionated whole breast irradiation and conventional standard fractionation of radiotherapy.

\section{Objectives}

Analysis of acute toxicities in hypofractionated radiotherapy versus conventional radiotherapy in early-stage breast cancer.

\section{MATERIALS AND METHODS}

This is a single institutional prospective non-randomised controlled trial done on patients with early breast cancer who underwent breast conservation surgery and required adjuvant radiation therapy in our hospital, which is a tertiary 
care cancer hospital in South India. In the radiation oncology outpatient department, 40 eligible early breast cancer patients who underwent breast conserving surgery with negative margins were enrolled in the study after taking their consent was taken for convenience. A total of 40 patients who fulfilled the inclusion criteria were divided into two groups with 20 in each arm by non-random method were included in the study who were treated in June 2014 - January 2016. 20 patients were chosen for Arm-A (Conventional Fractionation). The prescribed target dose is $50 \mathrm{~Gy}$ in 25 fractions at 200 cGy per fraction, 5 fractions a week by $6 \mathrm{mv}$ photons over 5 weeks followed by a sequential boost of $10 \mathrm{~Gy} / 5$ fractions over 1 week with electrons of appropriate energy to the tumour bed. Other 20 patients were chosen for Arm-B (Hypofractionation). The prescribed dose is $40 \mathrm{~Gy} / 15$ fractions at $2.67 \mathrm{~Gy} /$ fraction, 5 fractions a week by $6 \mathrm{mv}$ by photons over 3 weeks followed by a sequential boost of 12.5 Gy/ 5 fractions at $2.5 \mathrm{~Gy} /$ fraction over 1 week with electrons to the tumour bed. A detailed history and clinical examination is done. Patients were positioned on an AIO board (MEDTEC) in supine position with head turned to opposite side and arm abducted and externally rotated (90 deg. or greater) above the head. A 4 clamp thermoplastic (ORFIT) mask was made to cover the neck and chest. Our institute is equipped with Philips Big Bore CT scanner. Radio-opaque wires were used to delineate the scars. A planning Computed Tomography (CT) scan is done with CT slices of $5 \mathrm{~mm}$ thickness from below the mandible to the upper abdomen. The images were transferred to the treatment planning system station (XIO version 4.40.05). Contouring was done according to the Radiation Therapy Oncology Group (RTOG) breast contouring atlas for radiation therapy planning. The Clinical Target Volume (CTV) included the whole breast tissue and supraclavicular nodal regions in selected cases. It was expanded by $5 \mathrm{~mm}$ to create the Planning Target Volume (PTV). Organs At Risk (OARs) were contoured according to the RTOG guidelines. Lungs were contoured in the pulmonary window. Heart is contoured superiorly from the level of division of aortic arch and inferiorly till the apex of the heart. Oesophagus, spinal cord and contralateral breast were also contoured as OARs.

Boost is indicated in patient's cases who have high-risk features like age $<50$ yrs., high-grade tumours, node positive and lymphovascular invasion and perineural invasion positive. All patients were planned with forward planning Intensity Modulated Radiation therapy (IMRT) using two tangential with field-in-field approach. The boost volume was planned according to the post-operative changes seen on the planning CT (with $2 \mathrm{~cm}$ margin) using a direct electron field of approximate energy with a cone/ applicator. The quality of plans was verified using the Dose-Volume Histogram (DVHs) of the plan. The plan was accepted if all the constrains were achieved and $95 \%$ of PTV gets at least $95 \%$ of the prescribed dose, i.e. $\mathrm{PTV}_{95}>95 \%$.

\section{Assessment of Radiation Toxicity}

Patients were assessed for acute skin reactions weekly during the radiation therapy, at the completion of RT, after 1 month and 3 months after radiation therapy. The reactions were graded according to the RTOG acute toxicity grading criteria. The reported toxicity represents the maximal reported acute toxicity.

\section{Data Collection and Analysis}

- Data has been collected in case proforma and was entered in MS-XL 2007.

- $\quad$ SPSS-19.0 version has been used for analysing the data and MS-Word 2007 was used for reporting purpose.

\section{Statistical Analysis}

- Descriptive analysis of all the patients, disease related criteria, frequency of radiation reactions and grades among the study groups were analysed.

- All the qualitative factors were represented in the form of frequencies and percentages and the quantitative factors in the form of mean and standard deviations.

- The association between the categorised variables (Qualitative factors) was done using the Pearson's Chisquare test. The p-value reports are two-tailed and an alpha level of 0.05 is considered to be statistically significant.

- The influence of breast volumes on skin toxicities (Quantitative factors) -

- In CF group was done by using ANOVA test.

- In HF group was done by t-test.

\section{RESULTS}

The mean age of patients in CF arm was $46( \pm 6.9)$ yrs. and in the HF arm was $40.05( \pm 8.5)$ yrs. The pathology was mainly Invasive Ductal Cell Carcinoma (IDCC) in both the groups. 3 patients ( 2 in $\mathrm{HF}$ and 1 in $\mathrm{CF}$ arm) were having invasive lobular cell carcinoma and 1 patient was having medullary carcinoma (in $\mathrm{HF}$ arm). It was not statistically significant. Most of the patients in $\mathrm{CF}$ arm had well-differentiated tumours $50 \%(10 / 20)$ and moderately differentiated tumours $45 \%$ (9/20), while those in HF arm had poorly differentiated ones of $55 \%(11 / 20)$. It showed statistically significant value. The proportion of patients who had leftsided breast cancer is more in CF arm $60 \%(12 / 20)$ than in the HF arm 30\% (6/20). Many of the patients in HF arm had right-sided breast cancer. Most of the patients in both arms had T2 disease, 1 patient in HF arm had unknown tumour stage. The nodal status was almost evenly distributed between the two arms with majority of patients having N0 disease. The tumour stage and nodal status was not statistically significant between the two arms. Patients who had Hormone positive $\left[\mathrm{ER}^{+}\right]$breast cancer did not differ statistically between the two groups.

\section{Analysis of Skin Toxicity}

Patients were followed up to 3 months. In the CF group, 15 patients $(75 \%)$ had grade- 2 reaction and 3 patients $(15 \%)$ had grade- 3 reaction, while in HF group only 8 patients (40\%) had grade-2 reactions and none of them showed grade- 3 reactions. Most of the patients in the HF group had grade- 1 skin reactions (60\%), while in CF arm only 2 patients $(10 \%)$ had grade-1 skin reaction. None of the patients in both the arms showed grade- 4 skin reaction [Fig. 1]. There is a statistically significant difference between the two groups.

Breast volumes are measured by manually contouring the breast target volume, i.e. the clinical target volume-tumour (CTV-T). In the CF group, 3 patients who had grade-3 skin reaction had a mean breast volume of 1484.67 cc ( \pm 167 cc), 15 patients who had grade- 2 skin reaction had a mean breast volume of $956 \mathrm{cc}( \pm 219)$ and 2 patients who had grade- 1 skin 
reaction had a mean breast volume of 622 cc $( \pm 125 \mathrm{cc})$ [Fig. 2].

In the HF group, the mean breast volume for 12 patients who had grade-1 skin toxicity is $763.83( \pm 223)$ cc and for 8 patients who had grade- 2 skin toxicity is $1197.50( \pm 242.9)$ cc [Fig. 3].

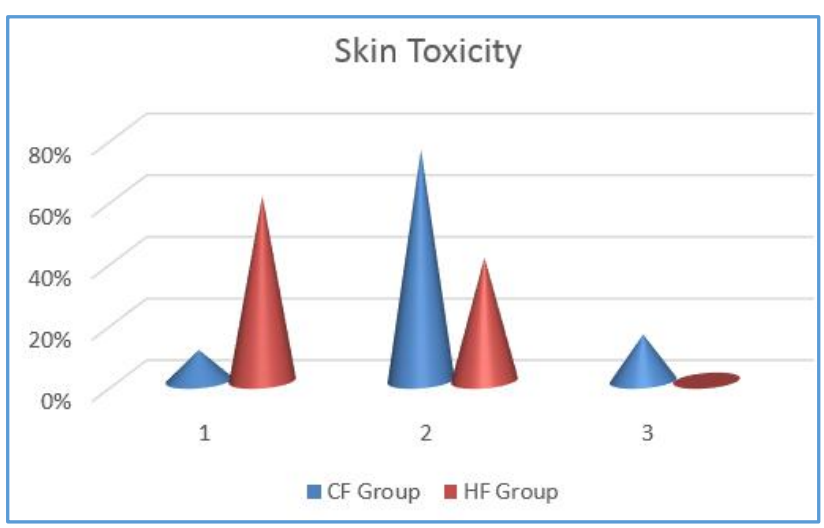

Figure 1. Bar Diagram showing Incidence of grades of Skin Toxicities in both Arms

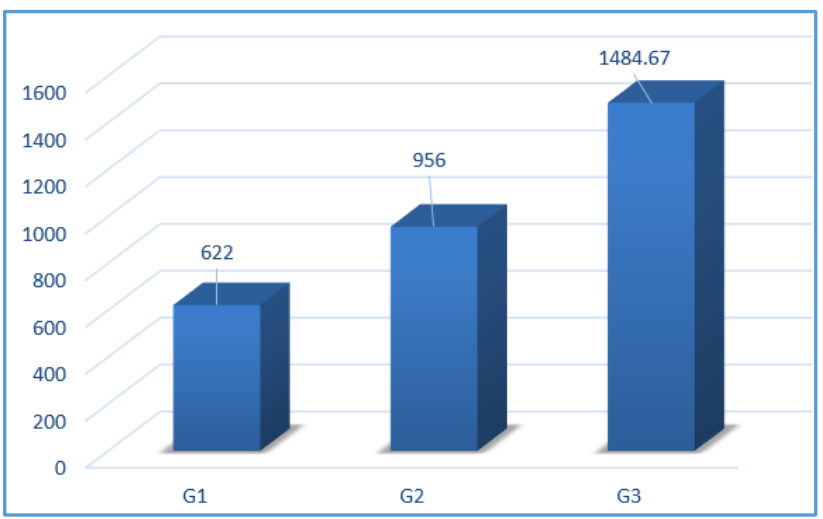

Figure 2. Bar Diagram showing Mean Breast Volumes in different grades of Skin Toxicities in CF Group

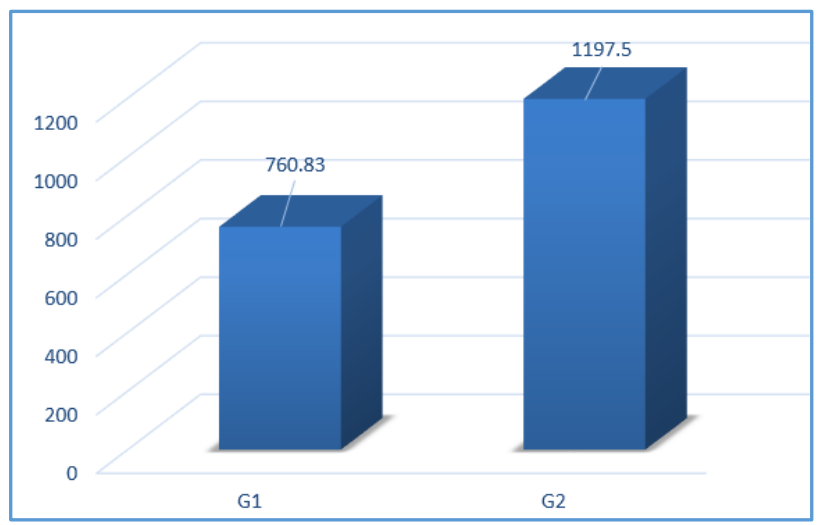

Figure 3. Bar Diagram showing Mean Breast Volumes in different grades of Skin Reactions in HF Arm

\section{DISCUSSION}

In the recent past, many efforts have been made to decrease the toxicities of EBRT after BCS like use of better immobilisation devices, breath holding techniques to decrease dose to the heart and lungs and practice of modern radiation techniques like 3D-CRT and IMRT.(1) Though these improve dose homogeneity, there is always some associated skin toxicity during or after RT. The individual's reaction of skin to radiotherapy depends on many factors, which are patient's tumour and/or treatment related.

In the present study, patients who received HF radiotherapy showed acceptable toxicity rate with only $40 \%$ incidence of grade-1 dermatitis. It is significantly lower than in patients treated with CF (75\% vs. $40 \%)$. This is consistent with the combined results from the START A and START B trials, where a change in skin appearance occurred significantly less often in the hypofractionated radiation arm (39 Gy and 40 Gy arms) when compared with the 50 Gy arm. $(2,3)$

A detailed evaluation of the results indicates that not all tested hypofractionated regimens are equally suitable for clinical use. Although, 39 Gy in 13 fractions was shown to be associated with less acute and late toxicity compared to conventionally fractionated radiotherapy, slightly increased ipsilateral breast cancer recurrences was observed in both trials (START Pilot and START A) testing this regimen. Consequently, 39 Gy in 13 fractions should not be preferentially used. The same applies for the use of $42.9 \mathrm{~Gy}$ in 13 fractions, since this schedule resulted in significantly increased late toxicity. The remaining schedules 40 Gy in 15 fractions, 42.5 Gy in 16 fractions and 41.6 Gy in 13 fractions are all suitable for routine clinical use; however, the most favourable observations were reported for the START B regimen. $40 \mathrm{~Gy}$ administered in 15 fractions of 2.67 Gy within 3 weeks resulted in significantly less acute and late toxicity and was associated with lower rates of ipsilateral breast recurrence. Since the 3 -week schedule is also more convenient for most patients, the START B regimen is the preferred schedule. The same regimen of 40 Gy in 15 fractions at 2.67 Gy per fraction for 3 weeks was used in the present study. $(2,3)$

In prospective randomised trials, the use of a tumour bed boost after whole breast irradiation reduced the risk of local recurrence even in patients with negative margins. $(4,5)$ Besides, an international survey showed that $85 \%$ and $75 \%$ American and European physicians respectively would deliver a boost even with negative margins. In this study, a sequential boost to the tumour bed is given to those patients who are $<50 \mathrm{yrs}$. of age or who have high grade, LVSI positive and node positive tumours with extensive DCIS component.

Despite the uniformity of the results, there are several differences in patient's selection, length of follow-up and use of systemic therapy and radiation boost among the three trials. In the Canadian study,(6) no patient received boost irradiation and only $10.9 \%$ received adjuvant systemic therapy. Furthermore, women with large breasts were excluded because of an increase in adverse cosmesis observed when such patients were treated with standard fractionation. In contrast in the START A and B trials, no exclusion criteria based on breast size were applied; $36 \%$ of patients received adjuvant systemic therapy and although its use was not standardised, most patients received a radiotherapy boost.(2,3) The American Society of Therapeutic Radiology and Oncology (ASTRO) guidelines reported that the HF-WBI was appropriate in patients of 50 years or older at diagnosis with pathological stage T1-T2 N0 disease treated with breast conserving surgery without chemotherapy and with a dose inhomogeneity on radiation plan $<7 \%$.

In the present study, there was a significant co-relation between breast volume and acute skin toxicity. Larger breast 
volume patients developed significantly more erythema G1 and G2 compared to smaller breast volume patients. Vicini and colleagues could show comparable results. Patients with breast volumes $>1600 \mathrm{cc}$ had more acute skin toxicity compared to those with breast volumes $<1000 \mathrm{cc}$.(7) Another study showed 27\% G2 erythema and 0\% G3 erythema in patients with breast volumes $<975 \mathrm{cc}$, while patients with breast volumes > $1600 \mathrm{cc}$ developed 59\% G2 and 3\% G3 erythema $(\mathrm{p}=0.002)$.

Fisher and colleagues summarised their data concerning breast volume and skin toxicity as follows: Patients with small breasts developed $\geq$ erythema G1 in $11-21 \%$, patients with medium sized breasts in $36-39 \%$ and patients with large breasts in $43-50 \%{ }^{(8)}$

Models of overdoses using the 40 Gy in 15 fractions regimens compared to $50 \mathrm{~Gy}$ in 25 fractions schedules predict that even for volumes that receive 1.5-times higher doses than prescribed, 40 Gy in 15 fractions is less critical for normal tissues as long as the fractionation sensitivity of the normal tissue can be described with an alpha/ beta ratio of $>1$ Gy. The lower 95\% limit of the alpha/ beta ratio for adverse effects regarding breast appearance calculated for the START A and B trials was $2.0 \mathrm{~Gy}$, indicating that large breast volume should not be an exclusion criterion for hypofractionated adjuvant radiotherapy in breast cancer. In addition, modern treatment planning including the use of intensity-modulated radiotherapy in breast cancer are able to limit the problem of overdoses in large breasts.

As the maximum dose is $<107 \%$ of the prescribed dose in all the patients in the present study, we could not find any specific correlation between maximum dose and acute skin toxicity. Chen and colleagues who demonstrated that larger volume receiving $>53.9$ Gy was a significant predictor of radiation induced skin toxicity.(9)

Patients with either large or small breast, the treatment modality did not affect the incidence of skin reactions. It is inferred that higher the breast volume, higher is the grade of acute skin reaction in both the groups.

\section{CONCLUSION}

The results in this study confirmed that HF-WBI is safe, which is in accordance with the data of large randomised trials. Therefore, moderated hypofractionated radiotherapy can be recommended as a standard treatment after BCS in majority of early-stage breast cancer patients. Hypofractionated radiotherapy provides a better quality of life, because it is a short duration treatment and shows comparable skin toxicities with that of Conventional Radiotherapy, more so if advanced treatment techniques like FIF 3D-CRT and IMRT are used.

\section{REFERENCES}

[1] Harsolia A, Kestin L, Grills I, et al. Intensity-modulated radiotherapy results in significant decrease in clinical toxicities compared with conventional wedge-based breast radiotherapy. Int J Radiat Oncol Biol Phys 2007;68(5):1375-80.

[2] The START Trialists' Group, Bentzen SM, Agrawal RK, et al. The UK of Breast Radiotherapy (START) Trial A of radiotherapy hypofractionation for treatment of early breast cancer: a randomised trial. Lancet Oncol 2008;9(4):331-41.

[3] The START Trialists' Group, Bentzen SM, Agrawal RK, et al. The UK Standardisation of Breast Radiotherapy (START) Trial B of radiotherapy hypofractionation for treatment of early breast cancer: a randomised trial. Lancet 2008;371(9618):1098-107.

[4] Bartelink H, Horiot JC, Poortmans PM, et al. Impact of a higher radiation dose on local control and survival in breast-conserving therapy of early breast cancer: 10year results of the randomized boost versus no boost EORTC 22881-10882 trial. J Clin Oncol 2007;25(22):3259-65.

[5] Romestaing P, Lehingue Y, Carrie C, et al. Role of a 10Gy boost in the conservative treatment of early breast cancer: results of a randomized trial in Lyon, France. J Clin Oncol 1997;15(3):963-8.

[6] Whelan TJ, Pignol JP, Levine MN, et al. Long-term result of hypofractionated radiation therapy for breast cancer. N Engl J Med 2010;362:513-20.

[7] Vicini FA, Sharpe M, Kestin L, et al. Optimizing breast cancer treatment efficacy with intensity-modulated radiotherapy. Int $\mathrm{J}$ Radiat Oncol Biol Phys 2002;54(5):1336-44.

[8] Fisher J, Scott C, Stevens R, et al. Randomized phase III study comparing Best Supportive Care to Biafine as a prophylactic agent for radiation-induced skin toxicity for women undergoing breast irradiation: Radiation Therapy Oncology Group (RTOG) 97-13. Int J Radiat Oncol Biol Phys 2000;48(5):1307-10.

[9] Chen MF, Chen WC, Lai CH, et al. Predictive factors of radiation-induced skin toxicity in breast cancer patients. BMC Cancer 2010;10:508. 